\title{
The electro-optic effect for intense terahertz pulses
}

Accelerator Science and Technology Centre, STFC Daresbury Laboratory, Warrington, WA4 4AD, UK

\author{
Received: 14 December 2007/ \\ Revised version: 29 January 2008 \\ Published online: 13 March 2008 • C) Springer-Verlag 2008
}

ABSTRACT The $\chi^{(2)}$ interaction between an intense terahertz ( THz) pulse and a weak optical probe is addressed, and a general solution obtained for the situation of an undepleted $\mathrm{THz}$ wave. It is shown that the common description of a phase retardation proportional to the $\mathrm{THz}$ electric field strength breaks down in strong-field conditions, unless the $\mathrm{THz}$ field is suitably slowly varying. The solution is obtained from a consistent description of sum- and difference-frequency generation driven by the $\mathrm{THz}$ field, the input optical field, and all optical fields generated through the interaction itself. For an interaction between monochromatic $\mathrm{THz}$ and optical waves a minimum of four waves (three optical and one $\mathrm{THz}$ ) need be considered in the weak-field limit; higher numbers of waves are needed at higher fields, with the extent of the required optical spectrum dependent on field strength, phase matching, and interaction length.

PACS 42.65.Ky; 42.65.Re; 78.20.Jq

\section{1}

\section{Introduction}

The electro-optic (EO) effect is widely utilized for the coherent detection of terahertz $(\mathrm{THz})$ radiation. While its main application has been in detection for $\mathrm{THz}$ time domain spectroscopy, more recently it has also found application in diagnostics of relativistic bunches in particle accelerators [1-4], and in the measurement of coherent synchrotron radiation (CSR) [5] and coherent transition radiation (CTR) [6, 7] generated by relativistic electron beams. In the spectroscopy applications, where the $\mathrm{THz}$ pulse is produced through laser interactions (optical rectification or Auston switch) pulse energies are modest, with pJ pulse energies typical in oscillator-based systems and $\lesssim 1 \mu \mathrm{J}$ for amplified laser systems. However, there have been some recent demonstrations of high $\mathrm{THz}$ pulse energy through optical rectification, with pulse energies of $\sim 10 \mu \mathrm{J}$ reported [8]. Recently, accelerator sources have been reported [7] with $\sim 100 \mu \mathrm{J}$

Fax:+44-1925-603192, E-mail: s.p.jamison@dl.ac.uk pulse energies in near-single-cycle pulses, with focused field strengths estimated to be as high as $7 \times 10^{7} \mathrm{~V} \mathrm{~m}^{-1}$.

For the high field strengths now being produced by optical rectification, and even more so by accelerator sources, it becomes necessary to re-examine the applicability of the usual description of the electro-optic effect as being a simple phase retardation proportional to field strength. Here, we address the description of the electro-optic effect in this high-field regime. The approach of Gallot and Grischkowsky [9] and Jamison et al. [10] is taken, whereby the EO effect is derived from the $\mathrm{THz}$-optical sum- and difference-frequency mixing. In those earlier works the small-signal, or equivalently no pump depletion, approximations were employed. In the strong $\mathrm{THz}$ field situation considered here the approximation of negligible depletion in the optical beam becomes marginal, and is dropped. This results in an infinite system of coupled wave equations for describing the high-field problem; an analytic solution for a completely general optical beam is presented, within the assumption of a THz beam sufficiently intense that the depletion of the $\mathrm{THz}$ wave by the $\chi^{(2)}$ interaction is negligible.

\section{The system of wave equations}

In a given experiment or application the interaction of THz and optical waves is dependent on the propagation directions and crystal orientation, and on the $\mathrm{THz}$ and optical polarization states. For a specific propagation geometry such as the collinear $\langle 110\rangle$ normal incidence geometry used in standard $\mathrm{ZnTe}$ or $\mathrm{GaP} \mathrm{THz}$ detection, it is possible to find a principal axes coordinate system in which the $\mathrm{THz}$ wave (of specified polarization) will allow optical waves polarized along a principal axis direction to interact only with identically polarized optical waves [10-12]. Choosing such a coordinate frame of reference allows the interaction with general optical polarization states to be described simply through the combination of independent interactions on each principal axis component. Here, we solve the coupled optical-THz wave equations for an optical wave polarized along a principal axis direction, and hence no polarization indices need be given on the waves. The results can then be considered quite general as they can be simply applied to arbitrary polarization states through a simple decomposition of the required wave into the principal axis frame; this approach is also compatible with the 
Jones matrix formalism for describing additional polarization optics in a given experiment.

In setting up the system of wave equations, the electric field spectral envelopes $\widetilde{A}(\omega, z)$ are defined with respect to the electric field via $\widetilde{E}(\omega, z)=\exp [\mathrm{i} k(\omega) z] \widetilde{A}(\omega, z)$. In the following the $\mathrm{THz}$ waves are additionally signified through a subscript, $\widetilde{A}_{\mathrm{THz}}(\omega, z)$, while optical waves are implied in the absence of a subscript.

Within the slowly varying envelope approximation, the interaction of optical and $\mathrm{THz}$ waves via the $\chi^{(2)}$ nonlinearity can be written, in SI units, as [9, 10, 13]

$$
\begin{aligned}
& {\left[\frac{\partial}{\partial z}+\beta^{\mathrm{opt}}\right] \widetilde{A}(\omega, z)} \\
& =\frac{\mathrm{i} \mu_{0} \varepsilon_{0} \omega^{2}}{2 k(\omega)} \exp [\mathrm{i} k(\omega) z] \\
& \quad \times\left[\chi^{(2)}(\omega ; \Omega, \omega-\Omega) \exp \left[-\mathrm{i} k_{\mathrm{THz}}(\Omega) z-\mathrm{i} k(\omega-\Omega) z\right]\right. \\
& \quad \times \widetilde{A}_{\mathrm{THz}}(\Omega, z) \widetilde{A}(\omega-\Omega, z) \\
& \quad+\chi^{(2)}(\omega ;-\Omega, \omega+\Omega) \exp \left[\mathrm{i} k_{\mathrm{THz}}(\Omega) z-\mathrm{i} k(\omega+\Omega) z\right] \\
& \left.\quad \times \widetilde{A}_{\mathrm{THz}}^{*}(\Omega, z) \widetilde{A}(\omega+\Omega, z)\right]
\end{aligned}
$$

where the frequencies $\omega, \Omega$, and $\omega-\Omega$ are all $>0$, and we take the propagation wavenumbers $k, k^{\mathrm{THz}}$ to be real. The absorption coefficient for the optical frequencies is given by $\beta^{\text {opt }}$. Both sum- and difference-frequency mixing contributions are explicitly included, through the terms with $\widetilde{A}(\omega-\Omega)$ and $\widetilde{A}(\omega+\Omega)$, respectively. The simultaneous inclusion of sum- and difference-frequency mixing is required for consistency, as neither can be assumed to be dominant ab initio. Furthermore, both of these mixing processes are required to describe the usual electro-optic effect $[9,10]$. Equation (1) is extended to describe a continuum of $\mathrm{THz}$ frequencies, with the assumption that the $\mathrm{THz}$ wave is sufficiently strong as to be undepleted by the $\chi^{(2)}$ interaction;

$$
\begin{aligned}
& {\left[\frac{\partial}{\partial z}+\beta^{\mathrm{opt}}(\omega)\right] \widetilde{A}(\omega, z)} \\
& =\frac{\mathrm{i} \omega}{2 c \eta} \times \int_{-\infty}^{\infty} \mathrm{d} \Omega \chi^{(2)}(\omega ; \Omega, \omega-\Omega) \\
& \quad \times \exp \left[\mathrm{i} \Delta k(\Omega, \omega) z-\beta^{\mathrm{THz}}(\Omega) z\right] \widetilde{A}_{\mathrm{THz}}(\Omega) \widetilde{A}(\omega-\Omega, z) .
\end{aligned}
$$

In (2), the linear propagation losses of the $\mathrm{THz}$ wave are retained through an explicit $z$ dependence of the $\mathrm{THz}$ amplitude, with $\widetilde{A}_{\mathrm{THz}}(\Omega, z) \rightarrow \exp \left[-\beta^{\mathrm{THz}}(\Omega) z\right] \widetilde{A}_{\mathrm{THz}}(\Omega)$, where $\beta^{\mathrm{THz}}(\Omega)$ is the THz absorption coefficient. The optical phase refractive index $\eta=c k / \omega$ has also been introduced in (2). Both sum- and difference-frequency mixing have also been accounted for through the integration of $\Omega$ over both positive and negative frequencies, and the definition of the (real) phase mismatch as

$\Delta k(\Omega, \omega)= \begin{cases}\Delta k^{+}(\Omega, \omega), & \Omega>0 \\ \Delta k^{-}(\Omega, \omega), & \Omega<0\end{cases}$ with

$$
\begin{aligned}
\Delta k^{+} & =\Delta k(\Omega>0, \omega) \equiv k(\omega)-k(\omega-\Omega)-k_{\mathrm{THz}}(\Omega) \\
& \approx \Omega / v_{\mathrm{g}}^{\mathrm{opt}}-\Omega / v_{\varphi}^{\mathrm{THz}}, \\
\Delta k^{-} & =\Delta k(\Omega<0, \omega) \equiv k(\omega)-k(\omega+|\Omega|)+k_{\mathrm{THz}}(|\Omega|) \\
& \approx-\Omega / v_{\mathrm{g}}^{\mathrm{opt}}+\Omega / v_{\varphi}^{\mathrm{THz}}=-\Delta k^{+} .
\end{aligned}
$$

In (4) and (5), $v_{\mathrm{g}}^{\mathrm{opt}}$ and $v_{\varphi}^{\mathrm{THz}}$ are the optical group velocity and $\mathrm{THz}$ phase velocity, respectively. In the following it is not necessary to use the approximations for $\Delta k^{ \pm}$in terms of the optical group velocity, although it does lead to a simpler description and will often be valid for $\mathrm{THz}$ interactions. The approximate phase-matching symmetry $\Delta k^{+}=-\Delta k^{-}$ will rarely, if ever, be valid for much higher frequencies, such as frequency mixing of optical and mid-infrared waves. That this symmetry may be valid for $\mathrm{THz}$ frequencies, or even that both $\Delta k^{+}$and $\Delta k^{-}$need be considered, is what differentiates the $\mathrm{THz}$ high-field interaction from three-wave descriptions of sum-frequency or difference-frequency mixing. For notational compactness, the $\mathrm{THz}$ absorption can be included within the phase-mismatching term, through the definition of a complex phase mismatch $\Delta \widetilde{k}=\Delta k+\mathrm{i} \beta^{\mathrm{THz}}$.

Equations (1) and (2) describe a coupling between the optical wave at frequency $\omega$ and the pair of sidebands at $\omega \pm \Omega$. To provide a description for an intense $\mathrm{THz}$ interaction no assumptions are made on the relative intensities of the sidebands, and allowance for the sidebands themselves to evolve through the $\chi^{(2)}$ interaction with the THz wave is provided. This leads to consideration of a system of equations for $A(\omega, z)$ over a (possibly continuous) spectral distribution $\omega$. To address this system of wave equations spanning the sideband frequency region of arbitrary extent, the THz spectrum is discretized into a set of many uniformly distributed $\mathrm{THz}$ waves with frequencies

$\Omega \in\{\ldots,-2 \delta,-\delta, 0, \delta, 2 \delta, \ldots, n \delta, \ldots\}$,

where $\delta$ is the spacing of $\mathrm{THz}$ frequencies (and which may tend to zero). Any particular $\mathrm{THz}$ frequency is denoted by $\Omega_{n} \equiv n \delta$, where $n$ may be positive or negative. As the span of the optical spectrum is coupled through this discretized $\mathrm{THz}$ field, the optical frequency in similarly discretized, with the same frequency spacing, and denoted by $\omega \rightarrow \omega_{m}$. We therefore have a system of equations for $\left\{A\left(\omega_{m}, z\right)\right\}$ :

$$
\begin{aligned}
& {\left[\frac{\partial}{\partial z}+\beta^{\mathrm{opt}}\left(\omega_{m}\right)\right] \widetilde{A}\left(\omega_{m}, z\right)} \\
& =\mathrm{i} \frac{\omega_{m}}{2 c \eta} \times \sum_{n=-\infty}^{\infty} \exp \left[\mathrm{i} \Delta \widetilde{k}\left(\Omega_{n}, \omega_{m}\right) z\right] \chi^{(2)}\left(\Omega_{n}\right) \\
& \quad \times \widetilde{A}_{\mathrm{THz}}\left(\Omega_{n}\right) \tilde{A}\left(\omega_{m}-\Omega_{n}, z\right) \delta .
\end{aligned}
$$

In the notation of (7), it has been assumed that the $\chi^{(2)}$ frequency dependence is dominated by the $\mathrm{THz}$ frequency response, so that we can write $\chi^{(2)}\left(\omega_{m} ; \Omega_{n}, \omega_{m} \pm \Omega_{n}\right) \rightarrow$ $\chi^{(2)}\left(\Omega_{n}\right)$; if necessary this assumption can be dropped in the following analysis. This system of equations in the optical field can be written in matrix form as

$\frac{\partial}{\partial z} \underline{\boldsymbol{A}}(z)=\left[B \underline{\underline{\boldsymbol{M}}}(z)-\underline{\underline{\boldsymbol{\beta}}}^{\mathrm{opt}}\right] \underline{\boldsymbol{A}}(z)$, 
where $\underline{\boldsymbol{A}}(z) \equiv\left\{\widetilde{A}\left(w_{m}, z\right)\right\}^{\mathrm{T}}$, the matrix $\underline{\boldsymbol{M}}(z)$ contains the coefficients appearing in the summation of $(7), \boldsymbol{\beta}$ is a diagonal matrix of the optical absorption coefficients, and $B \equiv \mathrm{i} /(2 c \eta)$. As an interim step to solving this system of equations, the phase-matching exponential term is expanded in the Taylor expansion

$$
\begin{aligned}
\exp \left[\mathrm{i} \Delta \widetilde{k}\left(\Omega_{n}, \omega_{m}\right) z\right]= & 1+\mathrm{i} \Delta \widetilde{k}\left(\Omega_{n}, \omega_{m}\right) z+\ldots \\
& +\left[\mathrm{i} \Delta \widetilde{k}\left(\Omega_{n}, \omega_{m}\right) z\right]^{s} \frac{1}{s !}+\ldots
\end{aligned}
$$

and the 'convolution' matrices, which are independent of $z$, are defined as

$$
\begin{aligned}
\underline{\underline{\boldsymbol{M}}}^{(0)} & =\left\{M_{m n}^{(0)}\right\} \equiv\left\{\omega_{m} \chi_{m-n}^{(2)} A_{m-n}^{\mathrm{THz}}\right\} \delta, \\
\underline{\underline{\boldsymbol{M}}}^{(1)} & =\left\{M_{m n}^{(1)}\right\} \equiv\left\{\omega_{m} \chi_{m-n}^{(2)} A_{m-n}^{\mathrm{THz}} \mathrm{i} \Delta \widetilde{k}_{(m-n), m}\right\} \delta \\
& =\left\{M_{m n}^{(0)}\left[\mathrm{i} \Delta \widetilde{\boldsymbol{k}}_{(m-n), m}\right]\right\}, \\
& \vdots \\
\underline{\underline{\boldsymbol{M}}}^{(s)} & =\left\{M_{m n}^{(s)}\right\} \equiv\left\{\omega_{m} \chi_{m-n}^{(2)} A_{m-n}^{\mathrm{THz}}\left[\mathrm{i} \Delta \widetilde{k}_{(m-n), m}\right]^{s} \frac{1}{s !}\right\} \delta \\
& =\left\{M_{m n}^{(0)}\left[\mathrm{i} \Delta \widetilde{k}_{(m-n), m}\right]^{s} \frac{1}{s !}\right\},
\end{aligned}
$$

where the following notational shorthand has been introduced:

$$
\begin{aligned}
A_{n}^{\mathrm{THz}} & \equiv \widetilde{A}_{\mathrm{THz}}\left(\omega_{n}\right) ; \quad A_{m-n}^{\mathrm{THz}} \equiv \widetilde{A}_{\mathrm{THz}}\left(\omega_{m}-\omega_{n}\right) ; \\
A_{m} & \equiv \widetilde{A}\left(\omega_{m}\right) ; \quad A_{m \pm n} \equiv \widetilde{A}\left(\omega_{m} \pm \omega_{n}\right) ; \\
\Delta \widetilde{k}_{(m-n), m} & \equiv \Delta \widetilde{k}\left(\omega_{m-n}, \omega_{m}\right) ; \\
\chi_{m-n}^{(2)} & \equiv \chi^{(2)}\left(\omega_{m-n}\right) .
\end{aligned}
$$

Since the THz spectrum and optical spectrum are discretized on the same spacing, the notational distinction of $\Omega_{n}$ and $\omega_{n}$ has been dropped, and both identically denoted as $\omega_{n}$.

With these matrix definitions, and the Taylor expansion (9), the discretized wave equation (8) can be written as

$$
\begin{aligned}
\frac{\partial}{\partial z} \underline{A}= & -\underline{\underline{\beta}}^{\mathrm{opt}} \underline{\boldsymbol{A}}+B \underline{\underline{M}}^{(0)} \underline{\boldsymbol{A}}+z B \underline{\underline{\boldsymbol{M}}^{(1)}} \underline{\boldsymbol{A}}+z^{2} B \underline{\underline{M}}^{(2)} \underline{\boldsymbol{A}}+\ldots \\
& +z^{s} B \underline{\underline{M}}^{(s)} \underline{\boldsymbol{A}}+\ldots
\end{aligned}
$$

The solution to this matrix equation has a simple matrix exponential form

$$
\begin{aligned}
\underline{\boldsymbol{A}}(z)= & \exp \left[-z \underline{\boldsymbol{\beta}}^{\mathrm{opt}}+z B \underline{\underline{\boldsymbol{M}}}{ }^{(0)}+\frac{z^{2}}{2} B \underline{\underline{\boldsymbol{M}}}^{(1)}+\ldots\right. \\
& \left.+\frac{z^{s+1}}{s+1} B \underline{\underline{\boldsymbol{M}}}^{(s)}+\ldots\right] \underline{\boldsymbol{A}}_{0},
\end{aligned}
$$

where $\underline{A}_{0}=\underline{A}(z=0)$. Having found this solution from the expansion of the phase-matching exponential term, this expansion can be contracted with the aid of the relationships between $M_{m n}^{(0)}$ and $M_{m n}^{(s)}$ given in (12). From this contraction, the matrix exponential solution of (23), with the complete infinite series of the phase-matching contributions, can be written simply as

$$
\underline{\boldsymbol{A}}(z)=\exp \left[-z \underline{\underline{\boldsymbol{\beta}}}^{\mathrm{opt}}+\frac{\mathrm{i}}{2 c \eta} \underline{\boldsymbol{T}}(z)\right] \underline{\boldsymbol{A}}_{0},
$$

where

$$
\begin{aligned}
\underline{\underline{T}}(z) & =\left\{T_{m n}\right\} \equiv\left\{M_{m n}^{(0)}\left[\frac{\exp \left(\mathrm{i} \Delta \widetilde{k}_{(m-n), m} z\right)-1}{\mathrm{i} \Delta \widetilde{k}_{(m-n), m}}\right]\right\} \\
& =\left\{\delta \omega_{m} \chi_{m-n}^{(2)} A_{m-n}^{\mathrm{THz}}\left[\frac{\exp \left(\mathrm{i} \Delta \widetilde{k}_{(m-n), m} z\right)-1}{\mathrm{i} \Delta \widetilde{k}_{(m-n), m}}\right]\right\} .
\end{aligned}
$$

Equations (15) and (16) are a solution to the coupled wave equations for arbitrary optical input. By choice of a suitably fine sampling of the optical spectrum, the solution for the discretized systems given by (15) and (16) can be used directly for simulations of the strong-field electro-optic effect. Such an approach is used in the example calculations given below.

It is also possible to obtain a continuous analytical result by taking the limit of vanishing frequency spacing $\delta \rightarrow 0$ following an expansion of the matrix exponential solution. The lowest-order matrix multiplication gives rise to the optical field given by

$$
\begin{aligned}
\widetilde{A}(\omega, z)= & \widetilde{A}_{0}(\omega) \mathrm{e}^{-z \beta_{\mathrm{opt}}} \\
& +\frac{\mathrm{i}}{2 c \eta} \mathrm{e}^{-z \beta_{\mathrm{opt}}} \omega \int \mathrm{d} \omega^{\prime} \widetilde{A}_{\mathrm{eff}}^{\mathrm{THz}}\left(\omega-\omega^{\prime}\right) \widetilde{A}\left(\omega^{\prime}\right),
\end{aligned}
$$

where the effective $\mathrm{THz}$ field is defined as

$$
\widetilde{A}_{\mathrm{eff}}^{\mathrm{THz}}(\omega) \equiv \widetilde{A}^{\mathrm{THz}}(\omega) \chi^{(2)}(\omega)\left[\frac{\exp \left(\mathrm{i} \Delta \widetilde{k}\left(\omega, \omega^{\mathrm{opt}}\right) z\right)-1}{i \Delta \widetilde{k}\left(\omega, \omega^{\mathrm{opt}}\right)}\right] .
$$

A corresponding solution in the time domain can be obtained though Fourier transformation of the frequency-domain results; the equivalent time-domain result is

$$
A(t, z)=A_{0}(t) \mathrm{e}^{-z \beta_{\mathrm{opt}}}+\frac{\mathrm{i}}{2 c \eta} \mathrm{e}^{-z \beta_{\mathrm{opt}}} \frac{\mathrm{d}}{\mathrm{d} t}\left[A_{\mathrm{eff}}^{\mathrm{THz}}(t) A_{0}(t)\right] .
$$

The first-order solutions given by (17)-(19) are in agreement with the small-signal, or no-depletion, result of Jamison et al. [10]. A continuous solution for the higher-order terms can similarly be obtained. By way of example, from the expansion of (15) the second-order contribution to the optical field is defined as

$$
\underline{\boldsymbol{A}}^{(2)}(z) \equiv \frac{1}{2 !}\left(\frac{\mathrm{i}}{2 c \eta}\right)^{2} \underline{\boldsymbol{T}}^{2} \underline{\boldsymbol{A}_{\mathbf{0}}} .
$$

Explicit expansion of the matrix multiplication leads to

$$
\begin{aligned}
A^{(2)}\left(\omega_{m}, z\right) \equiv & A_{m}^{(2)}(z)=\frac{1}{2 !}\left(\frac{\mathrm{i}}{2 c \eta}\right)^{2} \sum_{n} T_{m n} \sum_{p} T_{n p}\left(A_{0}\right)_{p} \\
= & \frac{1}{2 !}\left(\frac{1}{2 c \eta}\right)^{2} \mathrm{i} \omega_{m} \sum_{n} \delta A_{\mathrm{eff}, m-n}^{\mathrm{THz}}\left(\mathrm{i} \omega_{n}\right) \\
& \times \sum_{p} \delta A_{\mathrm{eff}, n-p}^{\mathrm{THz}}\left(A_{0}\right)_{p}
\end{aligned}
$$


where the effective THz field amplitude $A_{\mathrm{eff}, m-n}^{\mathrm{THz}}$ is defined as in (18). In the limit of $\delta \rightarrow 0$, we obtain

$$
\begin{aligned}
\lim _{\delta \rightarrow 0} A^{(2)}\left(\omega_{m}, z\right)= & \frac{1}{2 !}\left(\frac{1}{2 c \eta}\right)^{2} \mathrm{i} \omega_{m} \int \mathrm{d} \omega_{n} \widetilde{A}_{\text {eff }}^{\mathrm{THz}}\left(\omega_{m}-\omega_{n}\right) \mathrm{i} \omega_{n} \\
& \times \int \mathrm{d} \omega_{p} \widetilde{A}_{\mathrm{eff}}^{\mathrm{THz}}\left(\omega_{n}-\omega_{p}\right) \widetilde{A}_{0}\left(\omega_{p}\right)
\end{aligned}
$$

A simpler form of (21) can be found for the equivalent secondorder solution in the time domain. Fourier transformation, with recognition of the convolution integrals and the identification of the transformation of $\mathrm{i} \omega$ with the time-derivative operation, leads to the time-domain equivalent of (21):

$A^{(2)}(t, z)=\frac{1}{2 !}\left(\frac{1}{2 c \eta}\right)^{2} \frac{\mathrm{d}}{\mathrm{d} t}\left[A_{\mathrm{eff}}^{\mathrm{THz}}(t) \frac{\mathrm{d}}{\mathrm{d} t}\left[A_{\mathrm{eff}}^{\mathrm{THz}}(t) A(t)\right]\right]$.

Whilst in practice the discretized solution will most likely be more appropriate for numerical simulation of given experimental conditions, in principle the above approach allows for the construction of a continuous solution from the continuous limits of higher-order terms in the expansion of (15).

\section{Physical interpretation of solution}

To provide a physical interpretation of the solution given by (15) and (16), it is useful to look at the limit of perfect phase matching. By taking $\Delta \widetilde{k}_{(j-i), j} \rightarrow 0$ in (16), so that $\underline{\underline{T}} \rightarrow$ $\underline{\underline{M}}^{(0)} z$, a perfect phase-matching solution

$\underline{\boldsymbol{A}}=\exp \left[z B \underline{\underline{M}}^{(0)}\right] \underline{\boldsymbol{A}}_{0}$

is obtained. As expected, the same perfect phase-matched solution can be found by setting $\Delta \tilde{k}=0$ in the initial system of equations, so that in place of (13) the matrix representation of the system of equations becomes $(\partial / \partial z) \underline{\boldsymbol{A}}=B \underline{\underline{M}}^{(0)} \underline{\boldsymbol{A}}$.

In this limit of perfect phase matching the series expansion in powers of $\underline{\boldsymbol{M}}^{(0)}$, or equivalently in THz field strength,

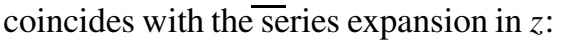

$\exp \left(z B \underline{\underline{\boldsymbol{M}}}^{(0)}\right)=1+z B \underline{\underline{\underline{M}}}^{(0)}-\frac{1}{2 !} z^{2} B^{2}\left[\underline{\underline{M}}^{(0)}\right]^{2}+\ldots$

The matrix exponential solution (23) can then be given the physical interpretation of a cascading of the interaction with generated sideband frequencies. When phase matching is included, the frequency-dependent elements in the matrix $\boldsymbol{T}$ must be considered to different orders in the distance $z$; despite this complication the cascading interpretation will remain valid.

To demonstrate the cascading interpretation a monochromatic $\mathrm{THz}$ wave with frequency $\Omega=\Omega_{r}$ is considered. The matrix $\underline{\boldsymbol{M}}$ will then have two off-diagonal rows of non-zero elements; i.e. $M_{m n}=0$ unless $n=m \pm r$. In Fig. 1 the structure of the matrix $\underline{\underline{M}}^{(0)}$ and its higher powers is shown for such a monochromatic wave. The solid diagonal line in each matrix schematic signifies the non-zero elements; as a visual guide the locations of the non-zero elements in the next lowest order term are shown as dotted lines. Under the matrix schematics is an energy level diagram schematic for the physical processes represented by the matrix terms. The interactions shown are, from left to right, the linear propagation; the generation of sidebands on the input optical wave; the generation of sidebands of the sidebands, which we term the second-order sidebands; and the generation of third-order sidebands. At each step in the cascading the sidebands are generated with a $\pi / 2$ phase shift. It is through this phase shift that the second-order sidebands that overlap the original input field are $\pi$-phase shifted with respect to that field, and give rise to depletion of the input optical field. In comparison to the result presented here, in the weak-signal approximation the expansion of (24), or more generally of (15), is truncated to first order in the $\mathrm{THz}$ field strength, with the effect that only the first two processes shown in Fig. 1 will occur.

To give an approximate estimate of the significance of the higher-order terms, the following numerical example is taken as indicative of realistic high-field experimental conditions: $\chi_{\mathrm{eff}}^{(2)}=r_{41}^{\mathrm{ZnTe}} \varepsilon^{2} \approx 320 \times 10^{-12} \mathrm{~m} \mathrm{~V}^{-1}[12,14], \omega \approx 2 \pi \times$ $375 \mathrm{THz}, \eta=3, A_{\mathrm{THz}}=10^{6} \mathrm{~V} \mathrm{~m}^{-1} \mathrm{THz}^{-1}$, and a propagation distance of $z=1 \mathrm{~mm}$. We therefore have $\max \left\{z B \underline{M}^{(0)}\right\} \approx$ 0.42 . Hence, the higher-order terms are indeed significant for $\mathrm{THz}$ field strengths that are now being accessed in accelerator laboratories. The relative magnitude of the cascaded $\chi^{(2)}$ effect can also be compared to the expected magnitude of the quadratic electro-optic effect, which is driven by the polarization $P^{(3)} \sim \chi^{(3)}\left(E^{\mathrm{THz}}\right)^{2} E^{\text {opt }}$. In the absence of reductions to the effective polarization through particular geometrical configurations, the magnitudes of the sidebands generated by the second-order cascaded $\chi^{(2)}$ and the first-order $\chi^{(3)}$ interactions will scale approximately as $\left[\left(\omega_{\text {opt }} / 2 c \eta\right) E^{\mathrm{THz}} \chi^{(2)} z\right]^{2} E^{\mathrm{opt}}$ and $\left(\omega_{\mathrm{opt}} / 2 c \eta\right)\left(E^{\mathrm{THz}}\right)^{2} E^{\mathrm{opt}} \chi^{(3)} z$, respectively. It follows that for the above parameters, and $\chi^{(3)}\left[\mathrm{m}^{2} / \mathrm{V}^{2}\right] \sim 10^{-8} \chi^{(2)}[\mathrm{m} / \mathrm{V}][15]$, the ratio of contributions
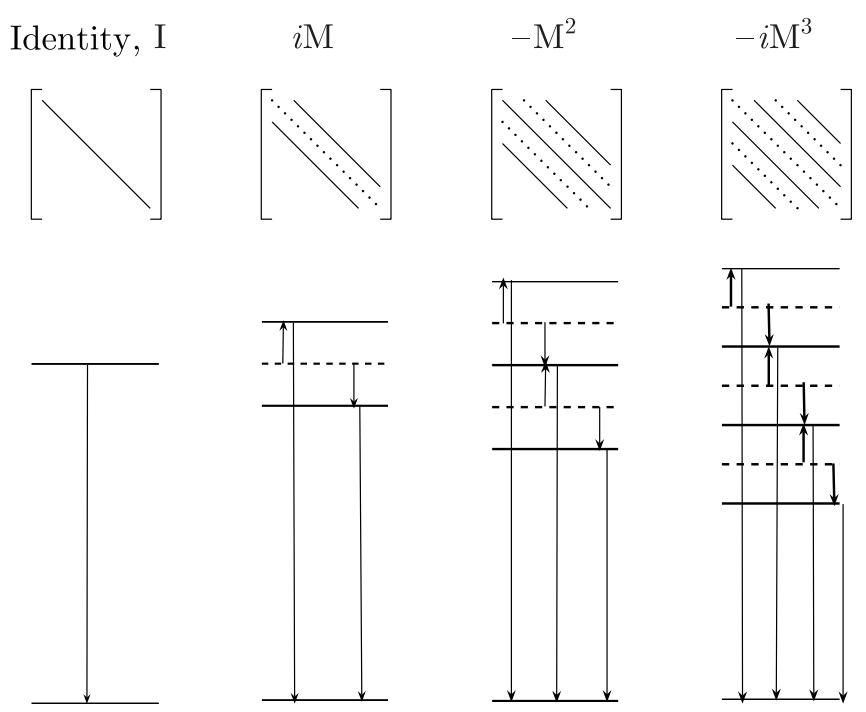

FIGURE 1 A summary of the physical processes associated with the individual terms in the matrix exponential Taylor expansion for a monochromatic $\mathrm{THz}$ wave. At the top is shown the matrix exponential expansion terms; the structure of the matrix for a monochromatic $\mathrm{THz}$ field is shown in the middle row. The lower row shows the particular frequencies generated by each successive interaction term; the levels shown as solid lines represent the final optical states, while the dotted levels are representing the connection to states obtained in the next lower order interaction 
in the $\omega_{\mathrm{opt}} \pm 2 \omega_{\mathrm{THz}}$ sideband will be dominated by $\chi^{(3)}$ only when $z \lesssim 50 \mu \mathrm{m}$; for the field strengths considered above, and in the following examples, the $\chi^{(3)}$ interaction will then only be expected to dominate when the sidebands themselves are relatively insignificant.

It is noted that for a dc ' $\mathrm{THz}$ ' field the matrix $\underline{\boldsymbol{M}}^{0}$ (or $\underline{\boldsymbol{T}}$ ) will be diagonal, and the sideband interpretation collapses. $\overline{\overline{I n}}$ that case the solution no longer provides coupling between the input optical frequencies. Each spectral component will have a solution of the form $\widetilde{A}=\widetilde{A}_{0} \exp \left(\mathrm{i} \alpha E_{\mathrm{dc}} z\right)$ which describes a phase retardation proportional to the field strength, and which holds regardless of the field magnitude. For a finitefrequency $\mathrm{THz}$ field such an identification between phase retardation and field strength cannot be expected to hold in general for all field strengths.

\section{Example calculations}

The solutions given by (15) and (16) have been evaluated for two example situations, the interaction of an intense quasi-monochromatic $\mathrm{THz}$ pulse with a quasimonochromatic optical pulse, and the interaction of a unipolar picosecond duration $\mathrm{THz}$ pulse with a broad bandwidth chirped optical pulse. The former serves to demonstrate the cascading of sidebands, and the influence of phase matching in suppressing the cascading to higher-order sidebands. The latter example represents the experimental conditions encountered for single-shot electro-optic characterization of relativistic electron beams, through the electro-optic detection of their THz-pulse-like Coulomb field profile [1, 2, 4], or of the emitted coherent synchrotron [5] or transition radiation $[6,7]$.

In Fig. 2 the interaction of a monochromatic $\mathrm{THz}$ pulse with a monochromatic optical pulse is shown. The THz pulse is taken to be a 14 ps FWHM duration pulse, with a central frequency of $1.5 \mathrm{THz}$ and a peak field strength of $5 \times 10^{5} \mathrm{~V} \mathrm{~m}^{-1}$. The optical spectrum following the electro-optic interaction with the THz pulse was calculated using (15) and (16), for two different interaction lengths of $1 \mathrm{~mm}$ and $3 \mathrm{~mm}$. In the evaluation of the matrix exponential solution the $\mathrm{THz}$ spectral range considered was $0-50 \mathrm{THz}$, while the optical spectral range evaluated was $375 \pm 50 \mathrm{THz}$. The discretized frequency spacing was $\delta=0.0242 \mathrm{THz}$, corresponding to a matrix $\underline{\boldsymbol{T}}$ with dimensions $4134 \times 4134$. With this matrix, the numerical solution of (15) was evaluated using algorithms in the Expokit package [16], run from within Matlab. In Fig. 2a and c, perfect phase matching has been assumed by taking $\Delta \widetilde{k}=0$, while in Fig. $2 b$ and $d$ the phase matching has been calculated using data for ZnTe, a commonly used electro-optic material. In the perfect phase-matching calculation the sidebands extend out to third and sixth orders in the short- and longerinteraction examples, respectively. Since in the case of perfect phase matching the interaction scales proportionally to the product of interaction length and field strength, an identical spectrum as that shown for the 3-mm interaction could be obtained for the $1-\mathrm{mm}$ interaction with a three-fold increase in field strength.

In the ZnTe phase-matching calculation, the growth of the higher-order sidebands during propagation has been inhibited; indeed, for higher $\mathrm{THz}$ frequencies where phase mis-
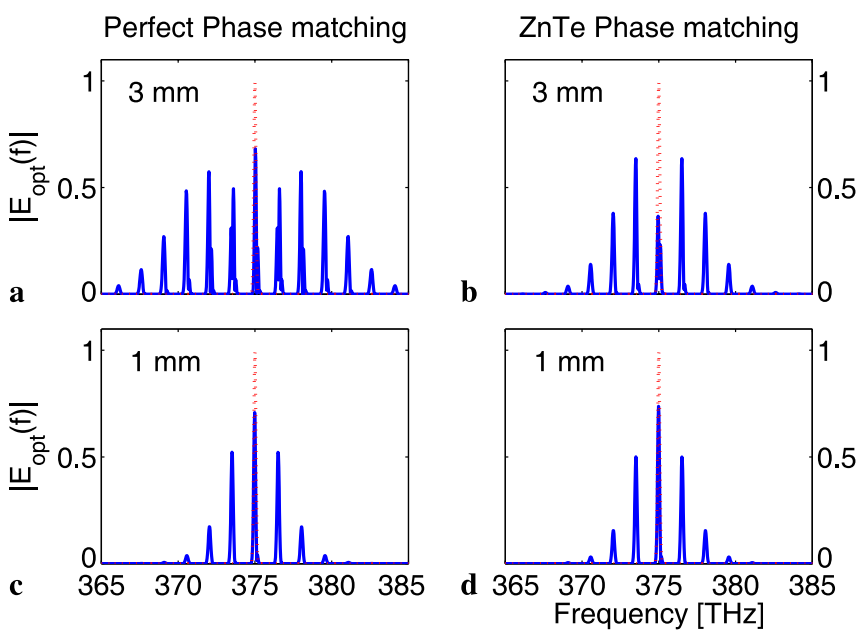

FIGURE 2 Optical spectra following the interaction with a quasi-monochromatic $\omega=1.5 \mathrm{THz}$ pulse, with a peak field strength of $5 \times 10^{5} \mathrm{~V} \mathrm{~m}^{-1}$, and for interaction lengths of $1 \mathrm{~mm}$ and $3 \mathrm{~mm}$; (a) and (c) assume perfect phase matching, while (b) and (d) include phase matching as expected for $\mathrm{ZnTe}$ material properties. In each plot, the dotted line is the input optical spectrum

match becomes larger (in ZnTe), the growth of sidebands beyond the first order can be completely suppressed. However, even for these higher frequencies, since the scaling of the interaction with field strength is no longer the same as for propagation distance, it remains possible to obtain higher-order sidebands with more intense field strengths over a shorter interaction distance.

If the weak-signal approximation was used to describe the same conditions used for the calculation shown in Fig. 2, we would obtain incorrect results in that (i) only the firstorder sidebands would appear, (ii) the optical fundamental would not be depleted, and (iii) the first-order sidebands would increase in size, as they are driven by the undepleted fundamental and also because they themselves are not subject to depletion from the interaction with the second-order sidebands.

Example calculations for the interaction of a unipolar $\mathrm{THz}$ pulse with a broadband chirped optical pulse are shown in Figs. 3 and 4. For these calculations, perfect phase matching was assumed to highlight the effects arising from the cascading of the $\chi^{(2)}$ interaction independently from the pulse distortion that arises from phase mismatch. The THz field was taken to be a unipolar Gaussian pulse with 2 ps FWHM. The input optical pulse had a Gaussian spectrum with (electric field) FWHM of $16 \mathrm{THz}$, centred at $375 \mathrm{THz}(\lambda=800 \mathrm{~nm})$. The chirp was described by a $\exp \left(\mathrm{i} \beta\left(\omega-\omega_{0}\right)^{2}\right)$ phase term, corresponding to a time-frequency correlation of $\Delta t=2 \beta\left(\omega-\omega_{0}\right)$. The magnitude of the chirp was $\beta=0.05 \mathrm{ps} /\left(\operatorname{rad~ps}^{-1}\right)$.

Figure 3 shows the spectral amplitude and phase retardation calculated for peak THz field strengths of $\left|E_{\mathrm{THz}}(t)\right|_{\max }=$ $10^{6} \mathrm{~V} \mathrm{~m}^{-1}$ and $4 \times 10^{6} \mathrm{~V} \mathrm{~m}^{-1}$, with a $1-\mathrm{mm}$ interaction length in $\mathrm{ZnTe}$, with the additional assumption of $\Delta \widetilde{k}=0$. The calculations are shown for both positive and negative polarities for the $\mathrm{THz}$ field. The retardation of any particular spectral sideband is determined by the field strength, by the polarity, and by the phase difference between the optical components and their sidebands. For a chirped pulse an asymme- 

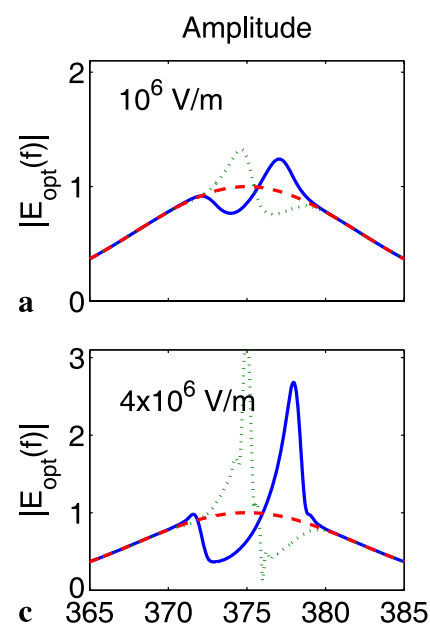
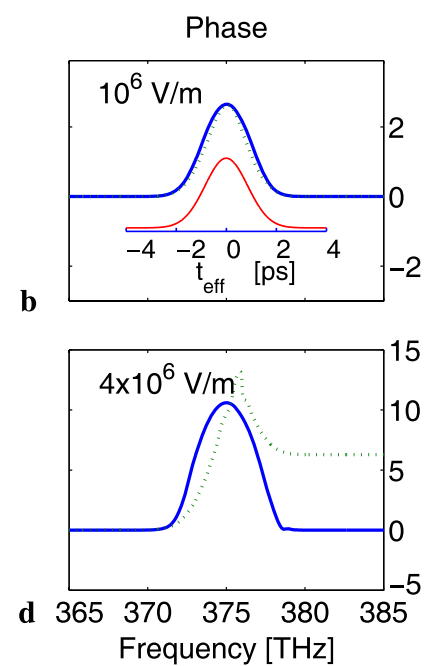

FIGURE 3 Spectral amplitude and phase change in a chirped optical pulse, induced by EO interaction with a unipolar THz pulse, 1 ps FWHM, with peak field strengths of $\pm 10^{6} \mathrm{~V} \mathrm{~m}^{-1}$ and $\pm 4 \times 10^{6} \mathrm{~V} \mathrm{~m}^{-1}$, and a $1-\mathrm{mm}$ interaction length in ZnTe. In (a) and (c) the spectrum of the input optical pulse is shown as a dashed line, while the spectra obtained after interaction with positive and negative polarity $\mathrm{THz}$ pulses are shown as solid and dotted lines, respectively. In (b) and (d) the phase changes $\left|\varphi-\varphi_{\text {ref }}\right|$ between the input (reference) pulse and the pulse after interaction with positive and negative polarity pulses are shown as solid and dotted lines. In (b) an effective temporal scale for the linearly chirped pulse is shown, together with the temporal profile of the $\mathrm{THz}$ pulse
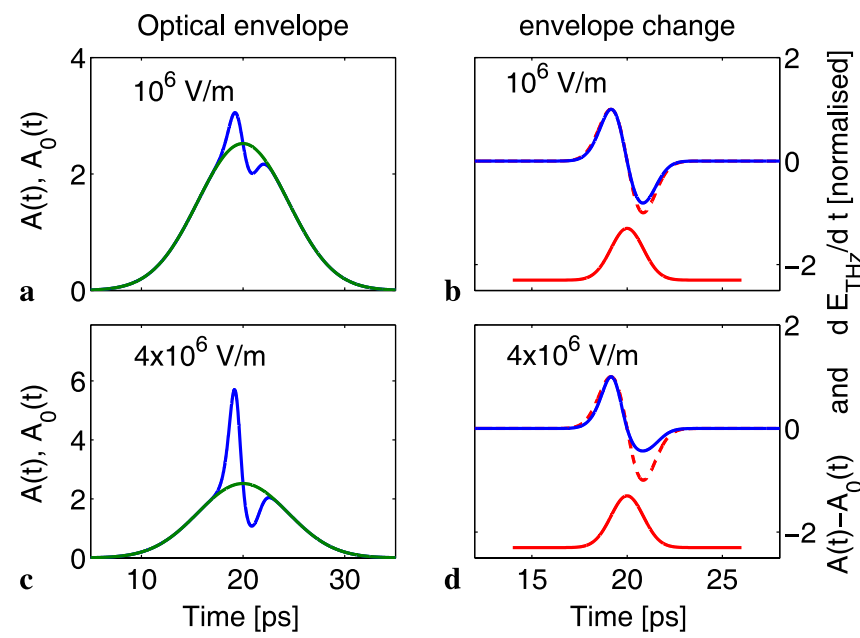

FIGURE 4 Temporal envelope and its change in a chirped optical pulse, induced by the same EO interactions as for Fig. 3. (a) and (c) show the optical envelope calculated with and without a $\mathrm{THz}$ pulse (blue and green, respectively). Only the calculation for positive polarity of the THz pulse is shown. In (b) and (d), the THz pulse profile $E_{\mathrm{THz}}(t)$, its time derivative $\mathrm{d} / \mathrm{d} t E_{\mathrm{THz}}(t)$, and the change in optical envelope $\Delta A_{\text {env }}(t)$ are shown (red, red-dashed, and blue lines, respectively). These plots are all normalized to allow comparison of the temporal variation

try in polarity is introduced as the $\chi^{(2)}$-induced retardation can be either additive or subtractive to the chirp-dependent phase difference between the probe frequency and the generated sidebands. An asymmetry also arises in the spectral amplitude, from the interference between the $\chi^{(2)}$-generated sidebands and the input chirped pulse. In Fig. $3 \mathrm{~b}$ the temporal profile of the THz pulse is also shown, where the $\Delta t=2 \beta(\omega-$ $\left.\omega_{0}\right)$ time-frequency correlation has been used in mapping the time-domain field into the spectral domain. For the low- field-strength calculation the phase retardation closely follows the temporal profile; this agreement is the basis of the single-shot $\mathrm{THz}$ detection technique of spectral decoding [1, 17,18]. For the high-field phase-retardation calculation of Fig. 3d the identification with the temporal profile no longer holds, and it is concluded that the spectral decoding concept is no longer valid in such high-field conditions. Complicated spectral modifications can arise from the high-field effects described here. High-field electro-optic $\mathrm{THz}$ measurements have been recently reported by Shen et al. [7] and spectral effects that are field-strength dependent were observed; however, their interpretation of the origin of the spectral features does not appear to take account of the high-field effects described here.

It is noted that in the calculations of Fig. 3 the relatively long $\mathrm{THz}$ pulse duration and the magnitude of the optical chirp were chosen to avoid observations of other artefacts known to occur in electro-optic spectral decoding for very short THz pulses $[10,19]$. The effects discussed above are strictly arising from the deviation of the $\chi^{(2)}$ interaction from the small-signal approximation.

Figure 4 presents the optical temporal profiles corresponding to the spectral calculations shown in Fig. 3. The optical field in the time domain $E(t)$ was determined directly from the calculated frequency-domain field, through Fourier transformation; the temporal envelope $A(t)$ was then evaluated from the oscillating field $E(t)$ through the absolute value of the analytic time domain field $E_{\mathrm{a}}(t)=E(t)+$ i $\mathscr{H}[E(t)]$, where $\mathscr{H}[E(t)]$ is the Hilbert transform of the field. In the low field strength limit, the change in the optical pulse in the time domain will, according to (19), be proportional to the time derivative $(\mathrm{d} / \mathrm{d} t)\left[A_{\text {eff }}^{\mathrm{THz}}(t) A_{0}(t) t\right]$. For a pulse with sufficiently small chirp, it follows that the phase modulation and amplitude modulation of the input field $A_{0}(t)$ arise predominantly from the $A_{\text {eff }}^{\mathrm{THz}}(t)(\mathrm{d} / \mathrm{d} t)\left[A_{0}(t)\right]$ and $(\mathrm{d} / \mathrm{d} t)\left[A_{\text {eff }}^{\mathrm{THz}}(t)\right] A_{0}(t)$ contributions, respectively. In Fig. 4b the change in the pulse envelope is shown, together with the time derivative of the $\mathrm{THz}$ pulse temporal profile; the change in envelope amplitude closely corresponds to the time derivative of the THz pulse, as expected. For the high-field calculation, shown in Fig. 4c and d, the temporal envelope is markedly simpler than the corresponding spectral information; perhaps surprisingly the envelope continues to closely follow the time derivative of the $\mathrm{THz}$ field, although some asymmetry is introduced in the magnitude of the positive and negative modulation.

\section{5}

\section{Conclusion}

The $\chi^{(2)}$ interaction of an intense $\mathrm{THz}$ pulse with a weak optical probe has been addressed, and a solution that generalizes the electro-optic effect to high-field conditions has been found. The solution has been obtained in a principal axes coordinate frame, for optical waves polarized along a principal axis. The solution can therefore be applied to arbitrary polarization states through appropriate combination of orthogonal principal axis components, together with any external polarization optics, using Jones matrix formalism. Together with such a Jones matrix formalism, the solution presented here can be applied to describing the electro-optic 
detection of $\mathrm{THz}$ pulses in the high-field regime, and the upconversion of $\mathrm{THz}$ radiation to the optical spectral region. While describing the high-field regime, the results also extend the description of the low-field regime to situations where the phase-matched interaction length is sufficiently long that higher-order sidebands or, equivalently, depletion of the optical fundamental, are not negligible. It is found that the direct interpretation of the electro-optic effect as giving rise to a retardation proportional to field strength may break down for high $\mathrm{THz}$ field strengths. However, as expected, for dc fields this interpretation will remain valid irrespective of field strength.

\section{REFERENCES}

1 I. Wilke, A.M. MacLeod, W.A. Gillespie, G. Berden, G.M.H. Knippels, A.F.G. van der Meer, Phys. Rev. Lett. 88, 124801 (2002)

2 G. Berden, S.P. Jamison, A.M. MacLeod, W.A. Gillespie, B. Redlich, A.F.G. van der Meer, Phys. Rev. Lett. 93, 114802 (2004)

3 A.L. Cavalieri, D.M. Fritz, S.H. Lee, P.H. Bucksbaum, D.A. Reis, J. Rudati, D.M. Mills, P.H. Fuoss, G.B. Stephenson, C.C. Kao, D.P. Siddons, D.P. Lowney, A.G. MacPhee, D. Weinstein, R.W. Falcone, R. Pahl, J. Als-Nielsen, C. Blome, S. Duesterer, R. Ischebeck, H. Schlarb, H. Schulte-Schrepping, Th. Tschentscher, J. Schneider, O. Hignette, F. Sette, K. Sokolowski-Tinten, H.N. Chapman, R.W. Lee, T.N. Hansen, O. Synnergren, J. Larsson, S. Techert, J. Sheppard, J.S. Wark, M. Bergh, C. Caleman, G. Huldt, D. van der Spoel, N. Timneanu, J. Hajdu, R.A. Akre, E. Bong, P. Emma, P. Krejcik, J. Arthur, S. Brennan, K.J. Gaffney, A.M. Lindenberg, K. Luening, J.B. Hastings, Phys. Rev. Lett. 94, 114801 (2005)
4 G. Berden, W.A. Gillespie, S.P. Jamison, E.A. Knabbe, A.M. MacLeod, A.F.G. van der Meer, P.J. Phillips, H. Schlarb, B. Schmidt, P. Schmüser, B. Steffen, Phys. Rev. Lett. 99, 164801 (2007)

5 S.P. Jamison, G. Berden, A.M. MacLeod, B. Redlich, A.F.G. van der Meer, D.A. Jaroszynski, W.A. Gillespie, Nucl. Instrum. Methods A 557, 305 (2006)

6 G. Berden, A.F.G. van der Meer, S.P. Jamison, B. Steffen, E.A. Knabbe, B. Schmidt, P. Schmüser, A.M. MacLeod, P.J. Phillips, W.A. Gillespie, Proc. EPAC 2006, 1058 (2006)

7 Y. Shen, T. Watanabe, D.A. Arena, C.C. Kao, J.B. Murphy, T.Y. Tsang, X.J. Wang, G.L. Carr, Phys. Rev. Lett. 99, 043901 (2007)

8 K.L. Yeh, M.C. Hoffmann, J. Hebling, K.A. Nelson, Appl. Phys. Lett. 90, 171121 (2007)

9 G. Gallot, D. Grischkowsky, J. Opt. Soc. Am. B 16, 1204 (1999)

10 S.P. Jamison, A.M. MacLeod, G. Berden, D.A. Jaroszynski, W.A. Gillespie, Opt. Lett. 31, 1753 (2006)

11 S.P. Jamison, A.M. MacLeod, G. Berden, D.A. Jaroszynski, W.A. Gillespie, Opt. Lett. 32, 1343 (2006)

12 S. Casalbuoni, H. Schlarb, B. Schmidt, P. Schmüser, B. Steffen, A. Winter, Numerical studies on the electro-optic sampling of relativistic electron bunches. TESLA Rep. 2005-1, available at http://tesla.desy.de (2005)

13 Y.R. Shen, The Principles of Nonlinear Optics (Wiley, New York, 1984)

14 N.C.J. van der Valk, T. Wenckebach, P.C.M. Planken, J. Opt. Soc. Am. B 21, 622 (2004)

15 L.R.C. Caumes, J.-P. Videau, E. Freysz, Phys. Rev. Lett. 89, 47401 (2002)

16 R. Sidje, ACM Trans. Math. Software 24, 130 (1998) [http://www.maths.uq.edu.au/expokit/]

17 J.A. Valdmanis, in Ultrafast Phenomena $V$, ed. by G.R. Fleming, A.E. Siegman (Springer, New York, 1986), p. 82

18 Z. Jiang, X.C. Zhang, Appl. Phys. Lett. 72, 1945 (1998)

19 S.P. Jamison, J. Shen, A.M. MacLeod, W.A. Gillespie, D.A. Jaroszynski, Opt. Lett. 28, 1710 (2003) 